\title{
Effect of Plateau Length on the Transformation of Internal Solitary Waves
}

\author{
Ming-Hung Cheng, Chih-Min Hsieh, Robert R. Hwang, and Shih-Feng Su
}

\begin{abstract}
The propagation and dissipation of internal waves over continental shelf bathymetry are complex phenomenon. The waveform would be re-generated while transmitting a submerged deep-shallow-deep topography. To study the effect of the marine topography on the evolution of an internal wave, numerical simulation is utilized to perform the flow evolution and waveform inversion of a large depression internal wave over a trapezoidal obstacle with different plateau. A finite volume based Cartesian grid method is adopted to solve the Reynolds averaged Navier-Stokes equations using a $k-\varepsilon$ model for the turbulence closure. Numerical results reveal that the re-generated waveform does not occur due to baroclinic wave. The shorter plateau length would induce strong vortex in back of the obstacle. Moreover, the wave amplitude, vorticity and turbulent kinetic energy are dissipated significantly. However, the level of the phenomenon decreases as the plateau length is larger than the wavelength.
\end{abstract}

Index Terms-Internal solitary wave, RANS equation, $k-\varepsilon$ turbulent model, flow field.

\section{INTRODUCTION}

Internal solitary waves (ISW) have been observed in the oceans and received attentions in the past decade. Strong ramification induced by the ISW not only influence on the marine ecology but also marine engineering works. The generation and propagating of ISW over shelf topography have been widely studied by theoretical analytics [1], [2], field observations [3], [4], laboratory experiments [5]-[7], and numerical simulations [8]-[10]. So far, it is well known that the ISW generated by the interaction of tidal currents in density stratified conditions and submarine ridges. Among the field measurements, the strongest ISW has been observed in the South China Sea (SCS) where its amplitude can be up to $170 \mathrm{~m}$ and velocity difference exceeds $2.6 \mathrm{~m} / \mathrm{s}$ between the upper and lower water layer [3]. Surprisingly, the ISW would remain as they propagate across various uneven seabed topographies without obvious energy damping. For example, the ISW in the northern SCS propagated across the continental shelf and existed in the vicinity of a submarine

Manuscript received December 16, 2015; revised February 26, 2016

Ming-Hung Cheng was with National Taiwan Ocean University, Keelung 20224, Taiwan. He is now with Department of System Engineering and Naval Architecture, Taiwan (e-mail: chengminghung@gmail.com).

Chih-Min Hsieh was with National Kaohsiung Marine University, Kaohsiung 80543, Taiwan. He is now with the Department of Maritime Information and Technology, Taiwan (e-mail: chihmin_623@yahoo.com.tw).

Robert R. Hwang was with National Taiwan Ocean University and Academia Sinica, Taipei 11529, Taiwan. He is now with Institute of Physics, Taiwan (e-mail: phhwang@gate.sinica.edu.tw).

Shih-Feng Su was with Tamkang University, Taipei 25137, Taiwan. He is now with Department of Water Resources and Environmental Engineering Taiwan (e-mail: stephen.shihfeng@gmail.com) sill with short plateau at Strait of Gibraltar [11], where the ISW encountered the topography with the sequence of water depth from deep to shallow plateau and then into deep water again.

Some reports have indicated that the self-generation of vortices with strong turbulent mixing within the water column and the waveform inversion across a continental shelf are two of the most important characteristics of an ISW propagation. Waveform transformation of the depression ISW in the ocean has been detected on satellite imageries [4]. Knickerbocker and Newell (1980) [2] used a transitional $\mathrm{KdV}$ to reveal that a depression ISW could transform into the elevation waveform once the upper layer depth is greater than the lower layer depth.

Due to the laborious works of the field measurements, laboratory experiments are extensively utilized to study the propagation of an ISW on pseudo slope-shelf topography with a stratified two-layer fluid. Temporal waveform evolutions associated with amplitude or energy at specific locations were measured from the results of video images and ultrasonic probes. Numerical modeling is adopted to investigate the evolution of density profiles, velocity field and waveform inversion. However, most of these numerical studies focused on the spatial or temporal process of an ISW on the front slope, and analyzed the temporal variations in waveform, velocity and wave energy. In addition, the evolution of waveform inversion during wave propagation over slope-shelf topography is limited.

The waveform would be re-generated as wave propagates beyond a trapezoidal obstacle, where the water depth becomes deeper. Cheng and Hsu (2010) [5] indicated that the plateau length may become a proxy to determine whether the inverted waveform could maintain its strength or be weakened swiftly. However, the effect of plateau length on the flow evolution of a depression ISW has not been investigated. In order to understand the effect of obstacle shape (including plateau length and shape) in the evolution of an ISW propagating over the submerged obstacle, this study uses a series of numerical simulations using RANS equations with the turbulence k- $\varepsilon$ model (Hsieh et al., (2015) [8]. The evolution of a depression ISW propagating over a trapezoidal obstacle with various plateau length and shapes are modeled by a transient two-dimensional, fully nonlinear, viscous flow phenomenon, upon solving the Reynolds averaged Navier-Stokes equations with a $k$ - $\varepsilon$ model for the turbulence closure in a fluid system with a pycnocline. In a two-dimensional Cartesian frame of reference, the physical domain is $12 \mathrm{~m}$ long ( $x$-axis) and $0.55 \mathrm{~m}$ deep ( $z$-axis), in which the fluid system consists of an upper and lower layer, and a pycnocline at the interface; while the physical conditions are the same as in [5]. The model results are 
analyzed to show the spatiotemporal evolutions of isopycnic, vorticity pattern, and turbulent kinetic energy. These modeling results provide better understanding into the effect of plateau length on waveform and flow evolution as a large depression ISW propagates over a submerged obstacle.

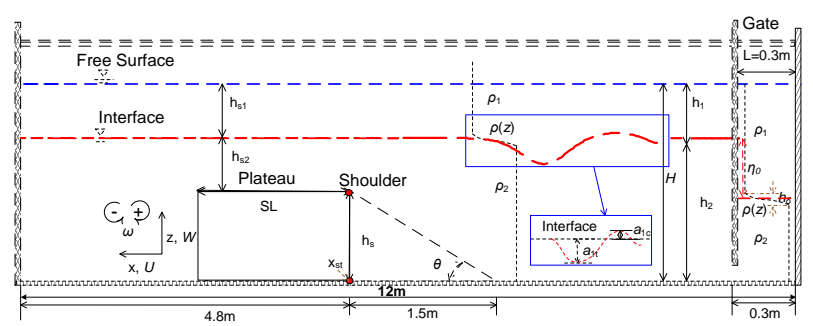

Fig. 1. Schematic diagram showing a depression ISW to be generated by gravity collapse mechanism. (Experimental condition: $h_{1}=0.14 \mathrm{~m} ; h_{2}=0.41$ $\left.m ; \eta_{0}=0.20 \mathrm{~m} ; h_{\delta}=0.04 \mathrm{~m} ; t_{0}=36 \mathrm{sec}\right)$.

\section{NumERICAL METHODOLOGY}

For studying the evolution of an ISW over a submerged trapezoidal obstacle in an incompressible free-surface flow with turbulent closure, conservation of mass and momentum is assumed for the fluid motion. Hence, a finite volume based Cartesian grid method is adopted to solve the general mass continuity equation and RANS using a $k-\varepsilon$ model for the turbulent closure. These governing equations are written as follows:

$$
\begin{gathered}
\nabla \cdot u=0 \\
\frac{\partial u_{i}}{\partial t}+u_{j} \frac{\partial u_{i}}{\partial x_{j}}=-\frac{1}{\rho} \frac{\partial P}{\partial x_{i}}+\nabla \cdot\left(v \frac{\partial u_{i}}{\partial x_{j}}-\overline{u_{i}^{\prime} u_{j}^{\prime}}\right)+g_{i} \frac{\Delta \rho}{\rho} \\
\frac{\partial \rho}{\partial t}+\nabla \cdot\left(\rho u_{i}\right)=\frac{\partial}{\partial x_{i}}\left(D_{m} \frac{\partial \rho}{\partial x_{i}}-\overline{u_{i}^{\prime} \rho}\right)
\end{gathered}
$$

where $u_{i}$ are the mean velocity components $(U, W)$ in a Cartesian coordinate systems $(x, z)$, respectively; $t$ is the time; $\rho$ is the density; $\Delta \rho$ is the density difference $\left(=\rho-\rho_{0}, \rho_{0}\right.$ is the density in upper layer); $\rho v$ and $D_{\mathrm{m}}$ is the molecular viscosity and molecular diffusivity, respectively; the item $-\overline{u_{i}^{\prime} u_{j}^{\prime}}$ is the Reynolds stresses; $-\overline{u_{i}^{\prime} \rho}\left(=\frac{v}{\mathrm{~S}_{\mathrm{ct}}} \frac{\partial \rho}{\partial x_{i}}\right)$ is the turbulent fluxes of the mean density $\rho ; g$ the gravitational acceleration; $S_{\mathrm{ct}}$ is the turbulent Schmidt number; The overbar (-) indicates the averaging of fluctuating quantities, and the Reynolds stresses, $-\overline{u_{i} u_{j}^{\prime}}$ are related to the corresponding mean rates of strain through the Boussinesq eddy viscosity model.

$$
-\overline{u_{i}^{\prime} u_{j}^{\prime}}=v_{\varepsilon}\left(\frac{\partial u_{i}}{\partial x_{j}}-\frac{\partial u_{i}}{\partial x_{i}}\right)-\frac{2}{3} k \delta_{i j}
$$

where $v_{\tau}$ is the turbulent viscosity, $\delta_{\mathrm{ij}}$ is the Kronecker delta, $k$ is the turbulent kinetic energy.

In order to close the system of mean flow equation, the $k-\varepsilon$ model is written in Eqs. (5) and (6).

$$
\frac{\partial k}{\partial t}+u_{j} \frac{\partial k}{\partial x_{j}}=\frac{\partial}{\partial x_{j}}\left(\frac{v_{t}}{\sigma_{k}} \frac{\partial k}{\partial x_{j}}\right)+P_{\varepsilon}+G-\varepsilon
$$

$$
\begin{aligned}
\frac{\partial \varepsilon}{\partial t}+u_{j} \frac{\partial \varepsilon}{\partial x_{j}}= & \frac{\partial}{\partial x_{j}}\left(\frac{v_{t}}{\sigma_{\varepsilon}} \frac{\partial \varepsilon}{\partial x_{j}}\right)+ \\
& C_{1 \varepsilon} \frac{\varepsilon}{k}\left(P_{\varepsilon}+C_{3 \varepsilon} G\right)-C_{2 \varepsilon} \frac{\varepsilon^{2}}{k}-R_{\varepsilon}
\end{aligned}
$$

where $P_{\varepsilon}, G$, and $R_{\varepsilon}$ are defined in Eqs. (7)-(9):

$$
\begin{gathered}
P_{\varepsilon}=v_{t}\left(\frac{\partial u_{i}}{\partial x_{j}}+\frac{\partial u_{j}}{\partial x_{i}}\right) \frac{\partial u_{i}}{\partial x_{j}} \\
G=\frac{g_{i}}{\rho} \frac{v_{t}}{S_{c t}} \frac{\partial \rho}{\partial x_{i}} \\
R_{\varepsilon}=\frac{C_{\mu} a^{3}\left(1-A / A_{0}\right)}{1+b A^{3}} \frac{\varepsilon^{2}}{k}
\end{gathered}
$$

In where $C_{\mu}=0.085 ; C_{1 \varepsilon}=1.44 ; C_{2 \varepsilon}=1.92 ; C_{3 \varepsilon}=0.2 ; \sigma_{\mathrm{k}}$ $=1.39 ; \sigma_{\varepsilon}=1.39 ; b=0.015$; and $A_{0}=4.38$ (An et al., 2012); $A$ is the ratio of turbulent time scale $(k / \varepsilon) ; S_{\mathrm{ct}}$ is the turbulent
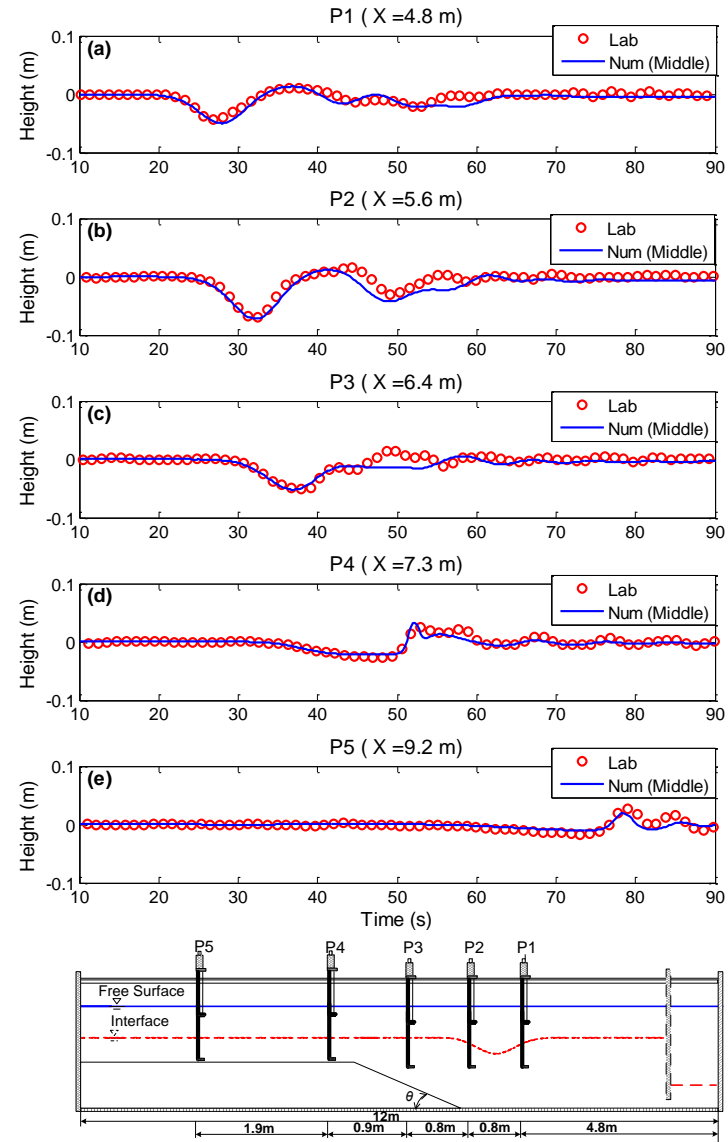

Fig. 2. Comparison between waveform calculated by numerical model and that of a laboratory experiment $(\mathbf{o})$ at Probes $1 \sim 5$.

All simulation runs start from a state of rest in the density stratified water $(U=W=0)$ with hydrostatic pressure utilized as pressure and density distribution over the entire computational domain. The interface level on either sides of the vertical gate called step depth $\left(\eta_{0}\right)$ is indicated on the right hand side (RHS) of the flume in Fig. 1, which is used to generated incident amplitudes for each ISW in depression. Following the gate rising at the speed of $0.25 \mathrm{~ms}^{-1}$, an ISW of depression is generated by the so-called collapse mechanism in the wave flume [12]. The boundary conditions are specified as a wall type. Noted the submerged obstacle is also adopted a fully reflected impermeable boundary. In addition, the boundary condition of free-surface displacement in the 
true volume-of-fluid (VOF) method is used to compute free surface motion and a fractional area/volume obstacle representation technique to model complex geometric regions. The numerical method employed is an improved version of the original VOF method, in which a second-order central difference scheme is used to discretize the continuity equation, pressure gradient, and diffusion terms. The convective terms are discretized using QUICK scheme with third-order accuracy. A semi-implicit method is adopted to evaluate the temporal terms while coupling between pressure and velocities remain implicit [8].

The verification run is carried out using the physical conditions of an existing laboratory case [5] with an long plateau. The waveform can be calculated by integrating the dimensionless vertical density difference between the initial time $(T=0 \mathrm{sec})$ and the present time $(T=t \mathrm{sec})$ to get the dimensionless ratio of average variations in ISW waveform. Obtaining the dimensionless ratio, the real average waveform is calculated by multiplying the maximum displacement $\left(\boldsymbol{h}_{\bar{\rho}}\right)$ of $\bar{\rho}\left(=\left(\rho_{1}+\rho_{2}\right) / 2\right)$ at the present time [8]. These results are presented in Fig. 2. This picture clearly shows the temporal variations of the leading waveform calculated numerically (blue dashed line, --) at probes P1 P5 are in good agreement with that of the laboratory experiment (red circle, o).

\section{RESULTS}

Based on the laboratory experiments [5], they showed sequential images to reveal that a depression ISW propagated over a trapezoidal obstacle with a short plateau length $(S L=$ $0.5 \mathrm{~m}$ ) or a long ones $(S L=4.8 \mathrm{~m})$. As a depression ISW propagates over a trapezoidal obstacle, the wave may propagate directly over deep-shallow-deep topography or may inverse an elevated IWs on the plateau and then pass across deep topography. Hence, two numerical results adopted that no plateau $(S L=0 \mathrm{~m}$, right triangle) and short plateau $(S L=0.5 \mathrm{~m})$ are plotted spatiotemporal evolutions of isopycnic with flow field, vorticity and turbulent kinetic energy (TKE) during this process in this section.
(1) $\mathrm{SL}=0$
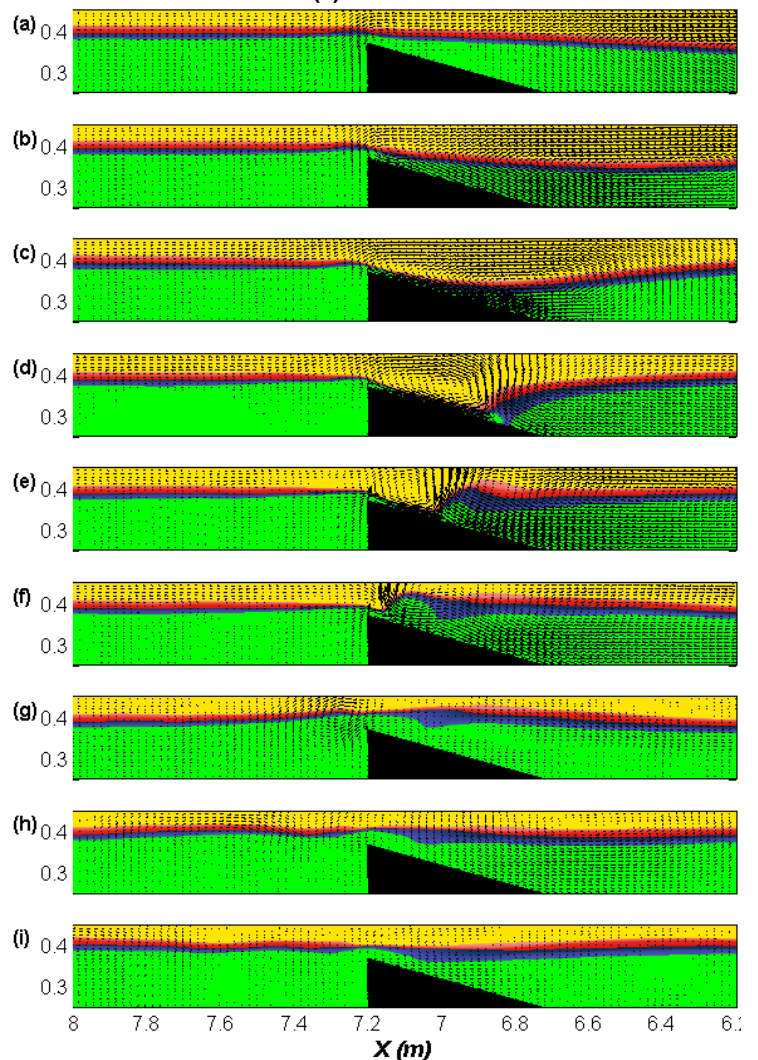

(2) $\mathrm{SL}=0.50 \mathrm{~m}$
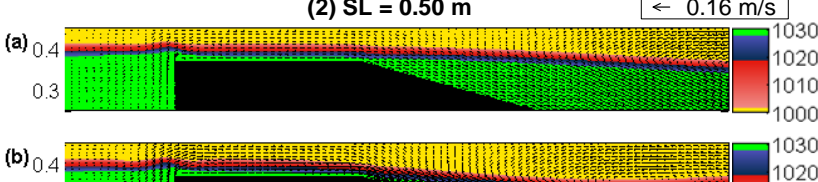

0.3
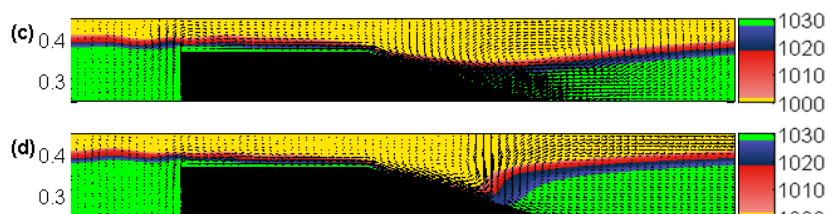

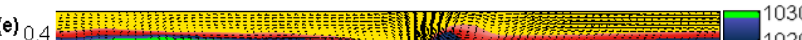
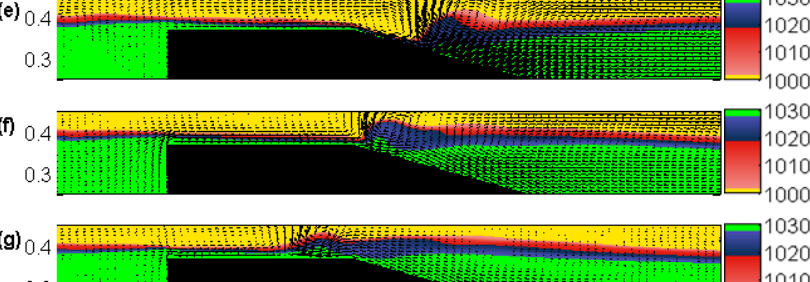

0
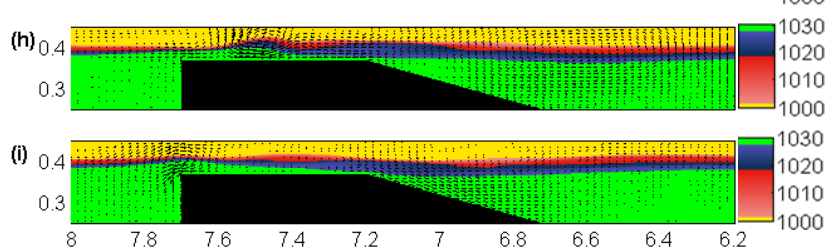

$x(m)$

Fig. 3. Numerical simulation for spatiotemporal isopycnic evolutions with field flow ( $S L=0.0$ and $0.5 \mathrm{~m}, T=34$ to $58 \mathrm{~s}$; snapshots are spaced apart by 3 sec)

\section{A. Spatiotemporal Isopycnic Evolutions with Flow Field}

By former numerical simulations, comparison between two cases of plateau length together with the computed isopycnic contours within the flow field are displayed in Fig. 3. Due to having the same front slope, when a depression ISW encounters a submerged trapezoidal obstacle with no or short plateau (Figs. 3(1)a-f and 3(2)a-f), the similar phenomena are follow as: firstly, the formation of an internal vortex rotating counterclockwisely is investigated before the leading trough of a depression ISW approaching the front slope of the obstacle (the flow field composes a forward velocity in the positive $x$-direction); And then, the strength of this vortex increases on the upper reach of the slope prior to the occurrence of an internal hydraulic jump in the rear of the leading waveform as the trough encountering the slope (Figs. $3(1) d$ and 3(2)d); Affected by gravity instability, the fluid on the horizontal plateau then commences debauching downward into the upper reach of the front slope; As the counterclockwise vortex reaches the shoulder of the plateau, the amplitude of the trough decreases significantly, and that of the wave crest increases, while the fluid draw-down 
continues across the shoulder (Figs. 3(1)f and 3(2)f). After the wave passes through the shoulder of the submerged obstacle, the different plateau length affects the waveform evolution. In the case of no plateau length $(\mathrm{SL}=0 \mathrm{~m})$, the transmitted waveform of leading trough quickly propagates in deep water but the wave crest decreases seriously due to uncomfortable depth (Fig. 3(1)g-i). In the meantime, a clockwise vortex induced by the wave-obstacle interaction also dissipates significantly in the rear of submerged obstacle. In the case of short plateau length $(S L=0.5 \mathrm{~m})$, the wave crest is formed gradually on the plateau but wave trough decreases again during the wave propagating on the plateau (Fig. 3(2)g). While the wave passes through the plateau (Fig.
$3(2) \mathrm{h}-\mathrm{i})$, the wave crest also decreases significantly in the rear of the plateau. In the meantime, a significant counterclockwise is investigated the left corner because of the interaction of like elevated ISW and the submerged obstacle (Fig. 3(2)h). Comparing with two results, the transmitted trough of leading wave in no plateau is larger than that in with short plateau. Although the like elevated IW is developed completely in having plateau length, the process induced serious dissipation of the wave crest in the rear of the trapezoidal obstacle. On the other hand, the transmitted amplitude of leading trough and crest in the former case is larger than that in the latter.
(1) $\mathrm{SL}=0$

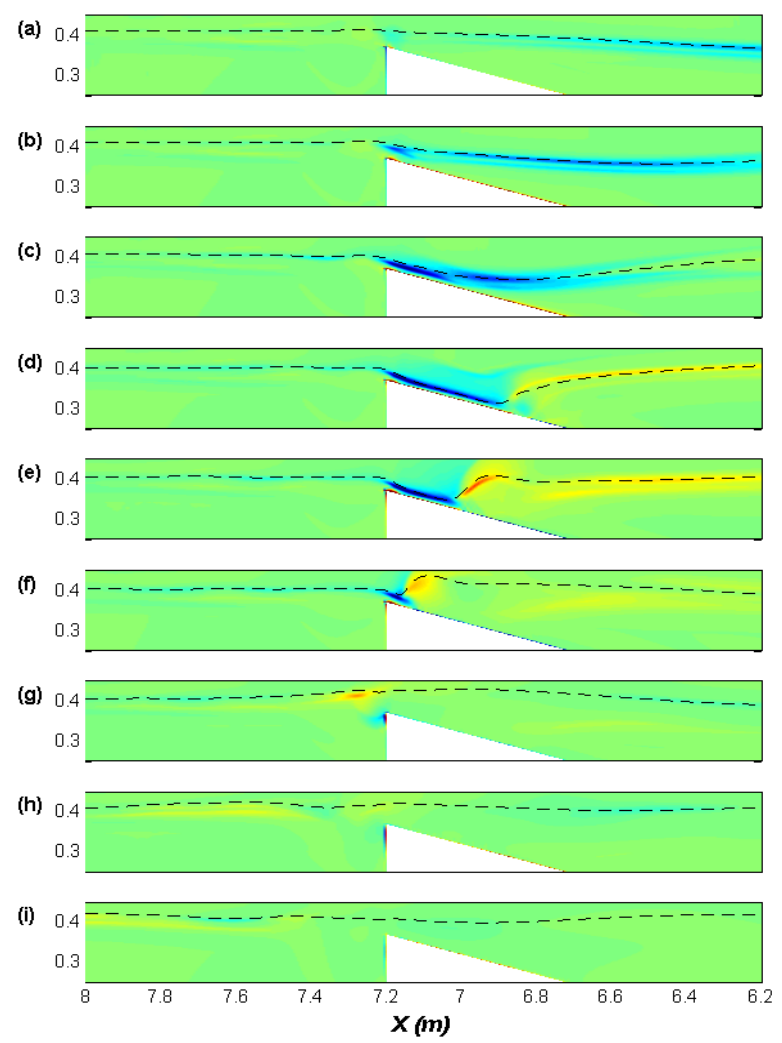

(2) $\mathrm{SL}=0.50 \mathrm{~m}$

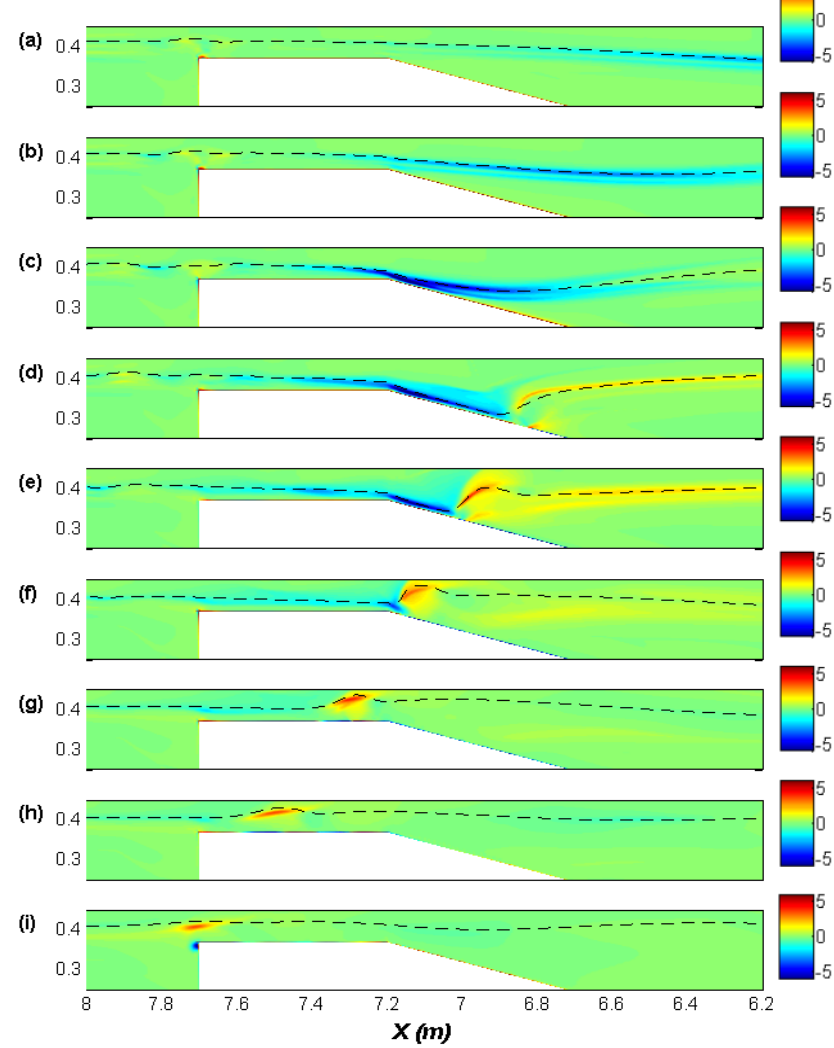

Fig. 4. Numerical simulation for spatiotemporal evolutions in vorticity contours ( $\mathrm{SL}=0.0$ and $0.5 \mathrm{~m}, \mathrm{~T}=34 \sim 58 \mathrm{~s}$; snapshots are spaced apart by 3 sec).

\section{B. Spatiotemporal Evolutions of Flow Velocity}

Beyond the waveform variations, flow evolutions are the other important view to investigate the process of an ISW propagating over submerged obstacle. Hence, the spatiotemporal evolutions of vorticity pattern ( $\omega=\frac{\partial W}{\partial x}-\frac{\partial U}{\partial z}, U$ and $W$ are horizontal and vertical velocity, respectively) are sketched in Fig. 4. Before the wave passes through the shoulder of the submerged obstacle, the evolutions of vorticity pattern in two cases are also similar due to same front slope. As an ISW approaching the front slope, negative vorticity for the counterclockwise vortex (blue bands) first dominates the entire physical domain (Figs. $4(1) a-b$ and 4(2)a-b), while the positive vorticity (for clockwise vortex, red band) is almost nonexistent. While the wave encounters the front slope, the positive vorticity is generated above the obstacle, as well as in the rear of the waveform (Figs. $4(1) \mathrm{c}-\mathrm{e}$ and $4(2) \mathrm{c}-\mathrm{e})$. In the meantime, the strength of the clockwise vortex (red bands) increases considerably because the narrowing space mentioned previously and the internal hydraulic jump in the upper reach of the front slope (Figs. 4(1)c-e and 4(2)c-e). At the same time, the original blue bands also vary swiftly due to shoaling and internal hydraulic jump (Figs. 4(1)d-e and 4(2)d-e). After it propagates over the shoulder, the negative vorticity (blue band) gradually reduces in two cases of no and short plateau (Figs. 4(1)f and 4(2)f). In the meantime, the positive vorticity increases slightly due to bottom friction and waveform inversion in the case with plateau (Fig. 4(2)f), but dissipates quickly because of unsuited depth in the case of no plateau (Fig. 4(1)f). As its propagating in deep water again (Fig. 4(1)g-i), the transmitted waveform (i.e. leading trough and crest) decreases gradually due to the water viscosity. If the wave propagates on the plateau (Fig. 4(2)g-h), the strength of the counterclockwise vorticity diminishes and that of the clockwise vorticity also decreases. After the wave propagates across the left corner, the clockwise vorticity dissipates 
quickly and induced a small counterclockwise vorticity in the rear of the plateau (Fig. 4(2)h-i). In order to compare clearly the difference between the plateau length while the wave encounters the front slope and passes through the obstacle, the enlarging pictures are shown in Fig. 5, together with the contours (noted blue dashed line is clockwise vortex; red line presents counterclockwise vortex). The strength of counterclockwise vortex (red line) in no plateau is weaker and shallower than that in short plateau (Figs. 5(1)d and $5(2) d)$. Beyond, clockwise vortex (blue line) is observed on the surface of the front slope. After the wave leaves the submerged obstacle, the strength of counterclockwise vortex induced by the leading crest in no plateau is smaller than that in short plateau length because of incomplete waveform in the former (Figs. 5(1)g and 5(2)i). On the other hand, the vorticity dissipation in the former is less than that in the latter during this process.
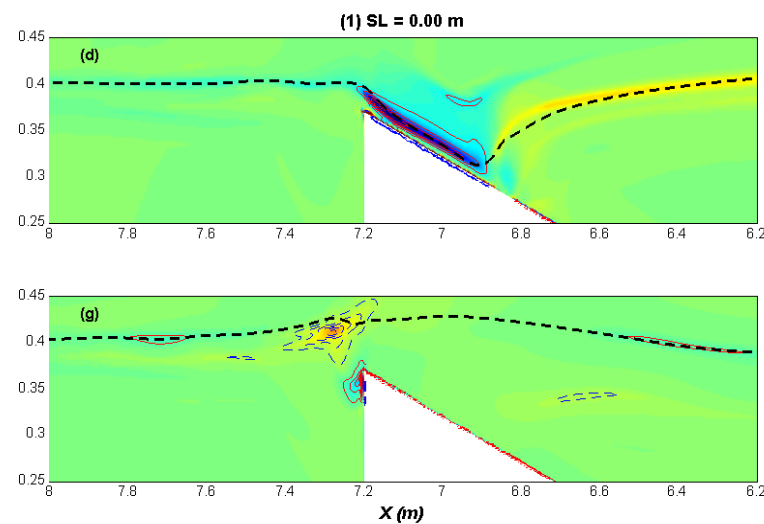

(2) $\mathrm{SL}=0.50 \mathrm{~m}$
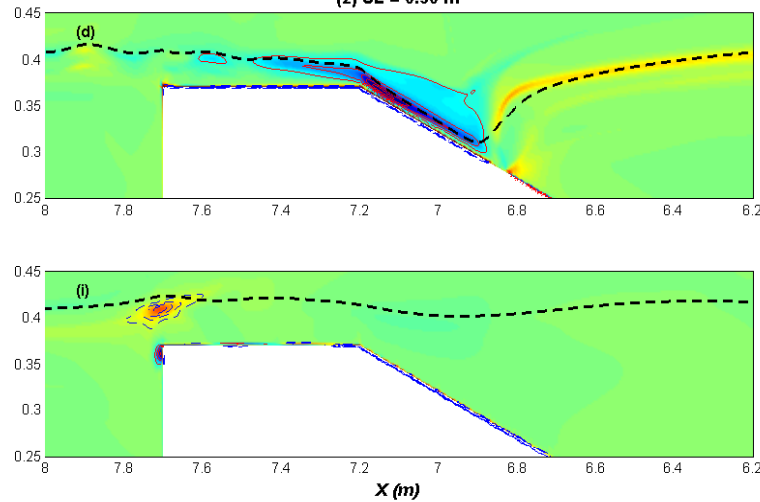

Fig. 5. Enlarging two significant temporal vorticity contours ( $\mathrm{SL}=0.0$ and $0.5 \mathrm{~m}, d=45 \mathrm{~s} ; g=52 \mathrm{~s} ; i=58 \mathrm{~s})$.

\section{Spatiotemporal Variations of Turbulent Kinetic Energy}

By using Eq. (5), local turbulent kinetic energy (TKE, $k$ ) is calculated and its spatiotemporal variations with two cases are illustrated in Fig. 6. Noted the strength of the TKE is highlighted using color bar, the interface in white dashed lines and the submerged obstacle in pink. In this figure, the evolutions of TKE are like the former two parameters and also similar before the wave passes through the shoulder (Figs. 6(1)a-f and 6(2)a-f). Before wave-obstacle interaction (Figs. 6(1)a-b and 6(2)a-b) and until the leading trough arrives at the upper reach of the front slope (Figs. 6(1)c and $6(2) c)$, the TKE of the leading trough is weak. As the wave approaches the front slope, the fluid originally residing above the plateau then debauches downward into the upper reach of the front slope, and the strength of the TKE grows rapidly with its size enlarging considerably (Figs. 6(1)d-f and
$6(2) d-f)$. While the wave is across the shoulder, the strength of the TKE dissipates quickly whether plateau length is no or short (Figs. 6(1)g-i and 6(2)g-i). Overall as shown in Fig. 6, it suffices to describe that the TKE induced by the original vortex is almost negligible comparing with that caused by wave-obstacle interaction on the upper reaches of the front slope, and a large amount of the TKE is dissipated near the shoulder no matter how long the plateau length is. During this process, the size of TKE induced by the wave-obstacle interaction in no plateau length is slightly larger than that in short plateau (Figs. 6(1)d-e and 6(2)d-e). That reason is the former evolutions has more water body in the rear of the obstacle. Although the size of TKE in the former is large slightly than that in the latter, the maximum of TKE in the former is less than that in the latter due to weaker wave-obstacle interaction.

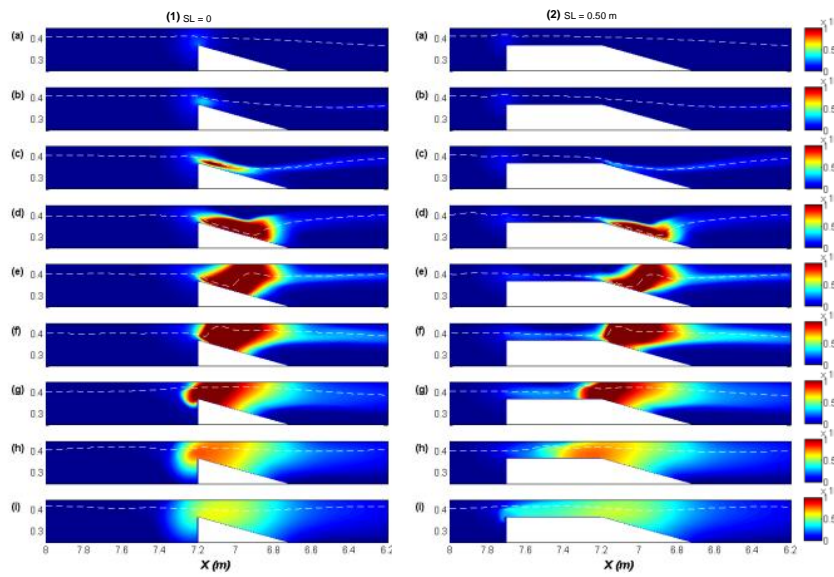

Fig. 6. Numerical simulation for spatiotemporal evolutions in turbulent kinitic energy ( $S L=0.0$ and $0.5 \mathrm{~m}, T=34 \sim 58 \mathrm{~s}$; snapshots are spaced apart by $3 \mathrm{sec})$.
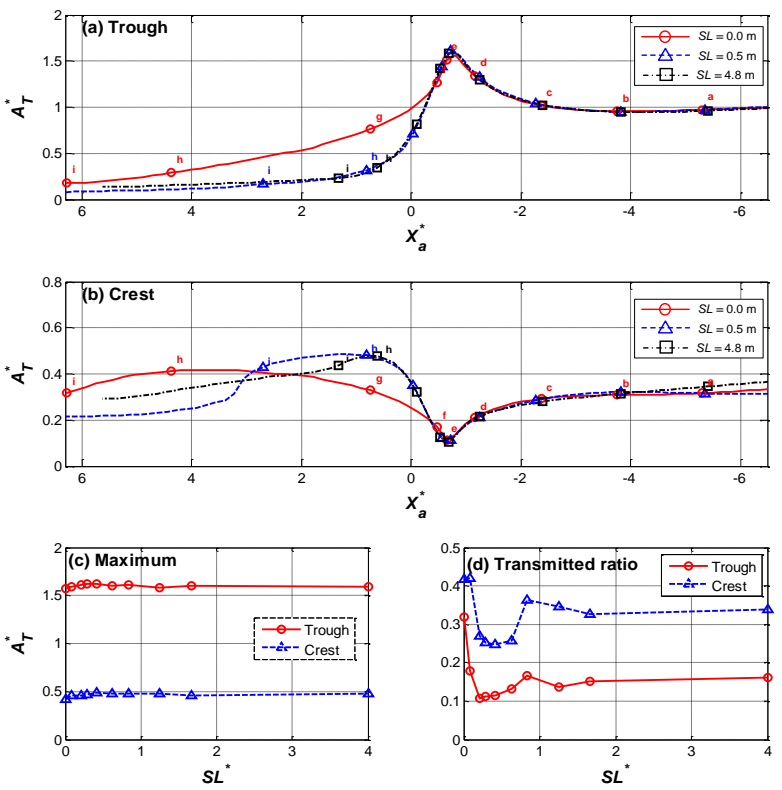

Fig. 7. the dimensionless transmitted amplitude versus the location of leading trough $\left(X_{\mathrm{a}}^{*}\right)$ : (a) the trough and (b) the crest; the dimensionless maximum amplitude versus the dimensionless plateau length $\left(S L^{*}\right)$ : (c) the spatiotemporal data and (d) the temporal data at $X_{\mathrm{a}}{ }^{*}=4$ (on the plateau).

\section{Discussions ON PERTINENT PROPERTIES}

By the former images of isopycnic, vorticity and TKE, the flow evolutions are shown generally in the section 3. Further 
discussions on the effect of plateau length (i.e., wave amplitude, velocity, vorticity, TKE and wave energy) are now given in terms of the dimensionless location from the leading trough $X_{\mathrm{a}}^{*}\left(X_{a}^{*}=\left(X_{a}-X_{s t}\right) / h_{s}\right)$ with all cases. The small flag in figures is the same time as the former diagrams (Figs. 3-4 and 6) and the RHS boundary of the abscissa at P1 in all figures is used as the initial position. Beyond the dimensionless spatiotemporal pictures, the maximum of these dimensionless parameters or transmitted ratio (the value at $X_{\mathrm{a}}{ }^{*}=4$ over incident value) are also plotted together with dimensionless plateau length $\left(S L^{*}=S L / l_{0}, l_{0}\right.$ is the incident wavelength $=1.20 \mathrm{~m}$ ).

\section{A. Spatiotemporal Isopycnic Variations with Flow Field}

Dimensionless amplitude of the leading trough or crest is defined to represent its variation during wave propagation,

$$
A_{r}^{*}=\left|\frac{a_{t}}{a_{0}}\right| \operatorname{or}\left|\frac{a_{c}}{a_{0}}\right|
$$

where $a_{0}(=0.051 \mathrm{~m})$ is the incident amplitude of the leading trough at P1, $a_{\mathrm{t}}$ and $a_{\mathrm{c}}$ are the local amplitude of the trough and crest, respectively.

Results for different plateau are plotted in Fig. 7, using $A_{T}^{*}$ versus $X_{a}^{*}$ and maximum $A_{T}^{*}$ or transmitted ratio versus $S L^{*}$, respectively. Due to the wave passes through the submerged obstacle, the wave speed in deep water is quicker than that on the plateau. Hence, the small flag in $S L=0.0 \mathrm{~m}$ is quicker than that in other cases (Fig. 7a-b). In Fig. 7a, the dimensionless transmitted amplitude of leading trough in three plateau length $(S L=0,0.5$, and $4.8 \mathrm{~m})$ has the same increased trend before the wave encounter the front slope $\left(X_{a}^{*}<-1\right)$ and decreases when the wave passes through the shoulder $\left(X_{a}^{*}>0\right)$. Due to no plateau effect, the decrease of leading trough in $S L=0 \mathrm{~m}$ is less than that in other cases. Beyond, the amplitude of leading trough is not re-generated when the wave propagates in deep water again. During the process, the transmitted value in short plateau length $(S L=$ $\left.0.5 \mathrm{~m}, X_{a}^{*}>3\right)$ is less than that in long length $(S L=4.8 \mathrm{~m}$, $\left.X_{a}^{*}>3\right)$. For the transmitted crest, the similar variations in three cases are found at $X_{a}^{*}>-1$ because of the same front slope. As the leading trough encounter the front slope and passes through the shoulder $\left(-1<X_{a}^{*}<2\right)$, the magnitude of $A_{T}^{*}$ in crest with short plateau length is larger than that with no plateau (Fig. 7b) because of small wave-obstacle interaction. However, the $A_{\tau}^{*}$ in crest with no plateau is larger than that with short plateau due to weak interaction in former. By comparing the short and long plateau length, the $A_{\tau}^{*}$ in crest with short plateau decreases significantly when the leading crest leaves the plateau and into the deep water $\left(X_{a}^{*}>3\right.$ in Fig. 7b). Choosing the maximum $A_{\tau}^{*}$ in trough and crest, the values of them have slight increase as the plateau length increases (Fig. 7c). Therefore, the plateau length affect weakly on the maximum values of leading trough. For the transmitted value ( $X_{a}^{*}=4$, in Fig. $7 d$ ), a significant value is found that the magnitude of the trough and crest decreases as the short plateau $\left(0<S L^{*}<1\right)$ and maintains a fixed value. Therefore, short plateau can induce the wave amplitude of leading trough and crest decreased seriously due to two significant wave-obstacle interaction (i.e., front slope and the left corner). As the plateau length is larger than the critical point, the intense decrease is weak gradually due to small crest-obstacle interaction. Moreover, the value of $A_{\tau}^{*}$ in no plateau is larger than that with plateau because of small obstacle interaction (Fig. 7d).

\section{B. Spatiotemporal Variations of Flow Vorticity}

To discuss the flow field variations during a wave-obstacle interaction, the quantity of local vorticity may serve as an important indicator. The dimensionless local clockwise vortex ( $\omega^{*}$ for positive vorticity) and counterclockwise vortex ( $\omega^{*}$ for negative vorticity) are defined as,

$$
\omega^{*}=100 *\left|\frac{\omega_{c w}}{U_{0} / H}\right| ; \omega_{\mathrm{avv}}^{*}=100 *\left|\frac{\omega_{c c w}}{U_{0} / H}\right|
$$

where $\omega_{c w}^{*}$ and $\omega_{c w}^{*}$ are associated with the local maximum clockwise and counterclockwise vortex, respectively, $H\left(=h_{1}\right.$ $+h_{2}$ ) is the total depth of the fluid in the flume, and $U_{0}$ is the maximum local horizontal velocity at P1. Like the dimensionless transmitted amplitude, four subplots which contain $\omega_{c w}^{*}$ and $\omega_{c \mathrm{cw}}{ }^{*}$ versus the dimensionless location from the leading trough $\left(X_{a}^{*}\right)$, maximum value of them and transmitted value at $X_{a}^{*}=4$ versus the dimensionless plateau length $\left(S L^{*}\right)$ are plotted together in Fig. 8. Before the wave propagates through the shoulder of a submerged obstacle with different plateau length, the strength of counterclockwise vortex (positive vorticity) in no plateau is less than that in short or long plateau length $\left(X_{a}^{*}<0\right.$ in Fig. $8 \mathrm{a})$. In the meantime, the strength of $\omega^{*}$ in short or long plateau length is similar $\left(S L=0.5\right.$ and $4.8 \mathrm{~m}, X_{a}^{*}<0$ in Fig $8 \mathrm{a})$. While the wave is across the shoulder, the strength of $\omega^{*}$ decreases significantly as the plateau length increasing $\left(X_{a}^{*}>\right.$ 0 in Fig. 8a) due to long wave-obstacle interaction. However, the variations of $\omega_{c w}^{*}$ is like as that of $\omega_{c c w}^{*}$ during this process. Due to more water body in the rear of the shoulder, the increase strength of $\omega_{c w}^{*}$ in no plateau is larger than that with plateau ( $X_{a}^{*}<0$ in Fig. 8 b). As the wave propagates over the plateau, the $\omega^{*}$ in short plateau is less than that in long plateau ( $X_{a}^{*}<3$ in Fig. 8 b) because of crest-obstacle interaction. By comparing the maximum of $\omega^{*}$ and $\omega^{*}$, the trend of $\omega_{c \mathrm{cw}}^{*}$ increases but that of $\omega_{c w}^{*}$ decreases as the plateau increases (Fig. 8c). At $X_{a}^{*}=4$, the transmitted ratio of $\omega_{c w}^{*}$ and $\omega^{*}$ (Fig. 8d) are not like as that of amplitude (Fig. 7d), 
especially at $\omega^{*}$. For the transmitted ratio of clockwise vortex $\left(\omega^{*}\right)$, the maximum value is occurred at $S L^{*}=0$, and then quick decreases as the plateau increases due to strong crest-obstacle interaction $\left(0<S L^{*}<0.5\right.$, Fig. $\left.8 \mathrm{~d}\right)$. As the plateau is larger than the critical length $\left(S L^{*} \sim 0.5\right)$, the $\omega^{*}$ increases gradually $\left(0.5<S L^{*}<1\right.$, Fig. 8 d $)$ because small incomplete crest induced by plateau effect propagates through the deep depth. While the complete crest is generated on the plateau $\left(S L^{*}>1\right.$, Fig. $\left.8 \mathrm{~d}\right)$, the strength of $\omega_{c w}^{*}$ decreases along with plateau length increasing. In Fig. 8d, the transmitted ratio of $\omega^{*}{ }_{c w}^{*}$ has a similar trend as that of $\omega_{c w}{ }^{*}$. However, the width of the concave in $\omega^{*}$ icw is narrow $\left(0<S L^{*}\right.$ $<0.5)$ and the decreased trend is larger than that of $\omega^{*}$ (i.e., the minimum value in $\omega^{*}$ is found in long plateau but that in $\omega_{c w}^{*}$ is observed in critical length). Hence, shortest plateau induces the strong vortex dissipation when a depression ISW nronagates nver a suhmeroed nhstacle
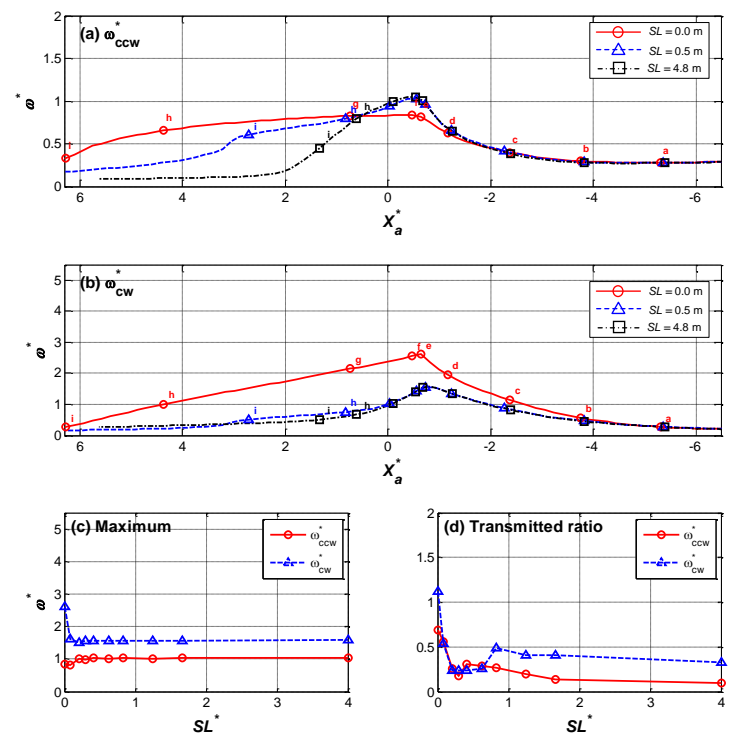

Fig. 8. the dimensionless transmitted vorticity versus the location of leading trough $\left(X_{\mathrm{a}}^{*}\right)$ : (a) the counterclockwise and (b) the clockwise; the dimensionless maximum vorticity versus the dimensionless plateau length $\left(S L^{*}\right)$ : (c) the spatiotemporal data and (d) the temporal data at $X_{\mathrm{a}}{ }^{*}=4$.

\section{Spatiotemporal Variations of Turbulent Kinetic Energy}

Based on the results calculated by Eq. (5), the turbulent kinetic energy (TKE, $k$ ) arising from the self-vortex motion within the fluid system due to wave-obstacle interaction is obtained. By normalizing its maximum value $\left(K_{\max }\right)$ with $u_{0}$, where $u_{0}$ is the initial velocity at P1, we can define the dimensionless maximum TKE $\left(K_{\max }^{*}=K_{\max } / u_{0}^{2}\right)$ for an ISW propagating over a trapezoidal obstacle. Upon plotting $K_{\max }^{*}$ against $X_{a}^{*}$ with different plateau length and maximum of $K_{\max }^{*}$ and transmitted ratio at $X_{a}^{*}=4$ versus dimensionless plateau length $\left(S L^{*}\right)$ are plotted in Fig. 9. It reveals that the variation in $K_{\max }^{*}$ is similar to that of the dimensionless counterclockwise vorticity (Fig. 8a), which has a peak when the leading trough is in the vicinity of the shoulder. And then, the strength of $K_{\max }^{*}$ decreases as the plateau length increases. In the short plateau, the strength of $K_{\text {max }}^{*}$ can obtain long time until the wave passes through the plateau ( $X_{a}^{*}>3$ in Fig. 9a). The maximum and the transmitted ratio of $K_{\max }^{*}$ have the same trend that decreases as the $S L^{*}$ increases because of long wave-obstacle interaction (Fig. 9b-c).
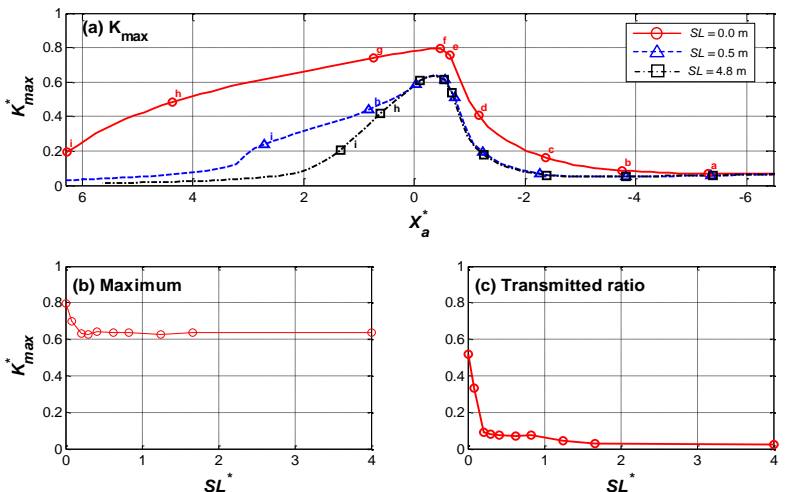

Fig. 9. (a) dimensionless transmitted TKE versus the location of leading trough $\left(X_{\mathrm{a}}^{*}\right)$; the dimensionless maximum vorticity versus the dimensionless plateau length $\left(S L^{*}\right)$ : (b) the spatiotemporal data and (c) the temporal data at $X_{\mathrm{a}}^{*}=4$.

\section{CONCLUSION}

In this paper, the numerical results revealed the spatiotemporal variations of amplitude, vorticity and TEK were highly depended on the plateau length. To study further the effect of obstacle shape and depth ratio, the numerical simulations and laboratory experiments will be compared to obtain the relative formula of the wave-obstacle interactions while an ISW propagates over variable topography.

\section{ACKNOWLEDGMENT}

The authors acknowledge the support from the Ministry of Science and Technology Taiwan, under the grants NSC 102-2221-E-019 -030 -MY3 and MOST 104-2811-E-019 $-002$.

\section{REFERENCES}

[1] R. Grimshaw, C. C. Guo, K. Helfrich, and V. Vlasenko, "Combined effect of rotation and topography on shoaling oceanic internal solitary waves," Journal of Physical Oceanography, vol. 44, no. 4, pp. 1116-1132, April 2014.

[2] C. Knickerbocker and A. C. Newell, "Internal solitary waves near a turning point,” Physics Letters A, vol. 75, no. 5, pp. 326-330, Feb. 1980

[3] C. Guo and X. Chen, "A review of internal solitary wave dynamics in the northern South China Sea," Progress in Oceanography, vol. 121, pp. 7-23, Feb. 2014.

[4] D. B. Reeder, T. F. Duda, and B. Ma, "Short-range acoustic propagation variability on a shelf area with strong nonlinear internal waves," Ocean 2008 - MTS/IEEE Kobe Techno-Ocean, vols 1-3, pp. 65-172, April 2008.

[5] M. H. Cheng and J. R. C. Hsu, "Laboratory experiments on depression interfacial solitary waves over a trapezoidal obstacle with horizontal plateau," Ocean Engineering, vol. 37, no. 8-9, pp. 800-818, June 2010.

[6] K. R. Helfrich and W. K. Melville, "On long nonlinear internal waves over slope-shelf topography," Journal of Fluid Mechanics, vol. 167, pp. 285-308, June 1986.

[7] T. W. Kao, F. S. Pan, and D. Renouard, "Internal solitons on the pycnocline - generation, propagation, and shoaling and breaking over a slope," Journal of Fluid Mechanics, vol. 159, pp. 19-53, Oct. 1985. 
[8] C. M. Hsieh, R. R. Hwang, J. R. C. Hsu, and M. H. Cheng, "Numerical modeling of flow evolution for an internal solitary wave propagating over a submerged ridge," Wave Motion, vol. 55, pp. 48-72, June 2015

[9] K. G. Lamb and A. Warn-Varnas, "Two-dimensional numerical simulations of shoaling internal solitary waves at the ASIAEX site in the South China Sea," Nonlinear Processes in Geophysics, vol. 22, no. 3, pp. 289-312, May 2015.

[10] T. Talipova, K. Terletska, V. Maderich, I. Brovchenko, K. T. Jung, E. Pelinovsky, and R. Grimshaw, "Internal solitary wave transformation over a bottom step: Loss of energy," Physics of Fluids, vol. 25, no. 3 , pp. 032110, March 2013.

[11] A. Vazquez, M. Bruno, A. Izquierdo, D. Macias, and A. Ruiz-Canavate, "Meteorologically forced subinertial flows and internal wave generation at the main sill of the Strait of Gibraltar," Deep-Sea Research Part I-Oceanographic Research Papers, vol. 55, no. 10 , pp. $1277-1283$, Oct. 2008.

[12] C. M. Hsieh, R. R. Hwang, J. R. C. Hsu, and M. H. Cheng, "Flow evolution of an internal solitary wave generated by gravity collapse," Applied Ocean Research, vol. 48, pp. 277-291, Oct. 2014.

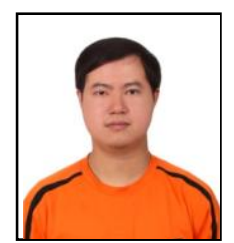

Ming-Hung Cheng was born in Taiwan. He is an ocean engineering with the master and Ph.D. degrees in the Department of Marine Environment and Engineering of National Sun Yat-sen University. He is currently a postdoctoral fellow in the Department of System Engineering and Naval Architecture of National Taiwan Ocean University. His research includes laboratory experiments/computed fluid dynamics for wave-obstacle/structure interaction.

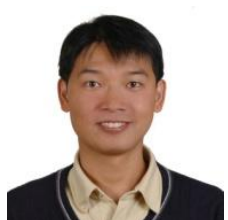

Chih-Min Hsieh was born in Taiwan. He is an ocean engineering with the master and Ph.D. degrees in the Department of Hydraulic and Ocean Engineering, National Cheng Kung University. He is currently an associate professor in the Department of Department of Maritime Information and
Technology, National Kaohsiung Marine University. His research field includes computed fluid dynamics for wave dynamics, sediment transport and coastal engineering.

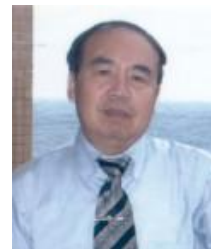

Robert R. Hwang was born in Taiwan. He is an engineering with the master degree in National Taiwan University and Ph.D. degrees in Iowa State University, America. He is currently a professor in the Department of System Engineering and Naval Architecture of National Taiwan Ocean University. His research field includes computed fluid dynamics for flow mechanism, turbulent diffusion and jet flow.

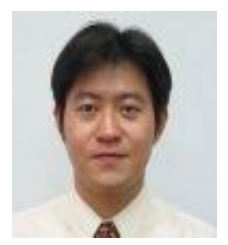

Shih-Feng Su was born in Taiwan. $\mathrm{He}$ is an engineering with the master degree in National Cheng Kung University and Ph.D. degrees in University of Florida, America. He is currently an assistant professor in the Department of Water Resources and Environmental Engineering of Tamkang University. His research field includes computed fluid dynamics for wave propagation and wave damping in reef. 